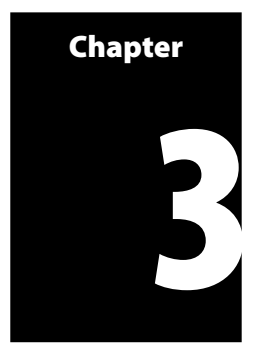

\title{
Returning to work experiences
}

\author{
Emily Johnson
}

The experience of returning to work in anaesthesia is different for each individual who undertakes the challenge. There is no escaping the fact it can be a daunting time, more so for some than others. In this chapter we include some personal reflections that have kindly been shared with the aim of providing some help and reassurance to those returning to work. We also discuss the results of regional surveys investigating the return to work experience.

Returning to work for the first day was daunting. It seemed so long since I had been in the role of being an anaesthetist. I had attended an update course, ALS and an airway day to prepare myself as much as possible. The thought of having to complete the practical procedures worried me the most. In reality many of these were like riding a bike - they came back very easily. What I wasn't so prepared for was the feeling of being mentally slower. To this day I don't think that I actually was, but this took me far longer to overcome.

Katy, Anaesthetic StR

For me, I found it much easier to concentrate at work if I was confident in my childcare arrangements. Having a nanny who came to the house was worth its weight in gold. We didn't have to change plans if one of the children was ill or if we'd had a disturbed night and were running late. It cost quite a lot, but it meant the children were happier, they could go to what they wanted in the way of clubs and lessons. I actually had two nannies - one did 4 days a week - about 4048 hours, and the other did one day a week. Being a good employer, providing a decent car so the children were safe, and doing my best to accommodate what our nanny needed to do, led to a good long-term relationship and therefore good help when things were difficult with children or work.

Another useful learning point was just to be 'good enough'. Prior to being a mum I had rather let work expand into home time, not helped as my husband is a non-medical academic, so was also very busy with grant applications. It was good to be reminded that I am our children's only mum - lots of other people can deal with things at work. Go home on time, and leave work at work.

The same applied to my approach to being a mum. It's about bringing up a child so he or she achieves their potential, not making them do what you want. I've had four children and every one was different, with different needs and enthusiasms. I watch others making their children fit into 'routines' and being told 'no' and 'don't', and suspect that this produces more stress amongst the parents and less happiness in the children. Remember, only people who have children who breast feed for 10 minutes every 4 hours can write books about childcare. The rest of society have 
children with different parenting needs and therefore do not write books or spend time on chat lines - we're looking after and enjoying our children.

Nancy, Consultant Anaesthetist

I was concerned about many aspects of returning to work. Particularly being able to do the practical procedures and knowing about any major changes in practice since I had been off. In practice the practical skills returned very quickly but the major hurdle was a reduced level of confidence. This I feel was partly due to returning to work less than full time and actually spending less time at work. Also observing all my peers flourish into confident consultants whilst I was still battling through workplace assessments. I had no objective feedback indicating my perceived reduced confidence was a problem and in fact was described as confident in many a multi-source feedback. I also don't believe it made me any worse at my job, it just made it harder personally. It is for this reason I have chosen to work full time in my consultant role.

Emily, Consultant Anaesthetist

I have taken two periods of maternity leave during my anaesthetic training, and clearly remember how I felt about returning to work. I certainly had mixed emotions about leaving my children; anxiety about leaving them for the first time, logistics of arranging childcare and worrying about whether they were happy seemed consuming at times. I don't think I was alone in feeling the perennial guilt about returning to further my career, rather than become a "stay at home mother". However, I also relished the opportunity to exercise my brain again and to remind myself that I wasn't totally defined by my role as a mother, even though I see it as my most important job.

I felt unprepared for my first return to work. I had tried to keep up to date as much as possible and had taught on an ALS course and on a Primary FRCA revision course. No formal RTW documentation was in place then, and I had an overwhelming sense of not really knowing what I was doing. My main sources of support were the LTFT representative for my school of anaesthesia and a really wonderful job share partner, who guided me through everything, step by step.

Practical procedures were not a problem, drug doses returned quickly. However, my overall 'flow' took a while to return. I felt a need to double check the most minor of details, and generally felt rusty. I think I put an immense amount of pressure on myself to be the best mother I could be, and to be the best I could be at my job, rather than give myself time to adjust. I think it took me several months to feel comfortable at work and enjoy it!

Thankfully, second time around, I was able to manage things very differently. A better sense of perspective, more knowledge of the RTW procedure and experience of managing a family and a busy job made things easier. I used my KIT days, and managed to participate in a number of projects whilst on maternity leave. In addition, a formal RTW process was now established; this forward planning ensured my return to clinical practice was as smooth as possible. Once again, a close support network of fellow LTFT trainees was invaluable to me. I have now used this experience to advise other trainees in my role as LTFT Trainee Representative for my school of Anaesthesia.

Anna, Anaesthetic StR

I have had three returns to work; 2008, 2011 and 2014. Comparing the three, what is most striking is the increase in awareness and support for the return to work process. My first return to work would have involved me starting in a hospital I had never worked in before directly onto night shifts. Thankfully my wonderful LTFT slot-share partner and a seasoned LTFT trainee stepped in to help. I think the first time was more difficult as I was adjusting to being a working mother, whereas the second and third time, this was no longer so new. I definitely worried lots about practical procedures and about having to ask for help in situations where I felt I might have been expected to manage solo. In reality neither of these was an issue. I found that familiarity with 
practical procedures came back quickly and I reasoned that it was always better to ask for help or just to check something with someone first if I was unsure. I don't recall anyone ever minding that. My second return to work was well supported with a lovely Educational Supervisor who had experience of returning to work herself. I also did the GAS Again course and some keeping in touch days, which helped. My most recent return to work has definitely been the easiest. By the third time I had more faith that I would get back up to speed and I followed a return to work programme so it was more structured. I think what I have found most helpful is support from colleagues who have been in similar situations. Realizing that what I was feeling was completely normal was very reassuring and helped to allay some of the anxiety.

Emma, Anaesthetic SpR

Understanding how others prepared for, and coped with, the transition back into clinical anaesthesia should help those having to return after a career break. However, there are inevitably going to be some elements of the process that come as a surprise and that leave any preparation feeling inadequate. The resulting effect is an undermining of confidence at a time that is already likely to feel daunting. Awareness of the issues one is likely to encounter and preparation tailored to suit individual needs ${ }^{[1]}$ are the only solutions.

To establish the commonly encountered problems in returning to work there have been Return to Work (RTW) Surveys conducted in the West Midlands Local Education and Training Board (LETB) ${ }^{[2]}$. The first of these was completed for those trainees who returned to work between 2008 and 2012. It established many (>71\%) trainees had difficulties on returning to work from a period of $>6$ months leave and most felt that their RTW could have been improved. Commonly encountered problems included paperwork for less than full time (LTFT) training, pay issues, childcare difficulties and rota issues including inadequate supervision and on-call shifts being allocated too soon. There was a general lack of awareness of keeping in touch (KIT) days and RTW courses. The length of time reported to regain confidence varied from a few days to 6 months. This was noted particularly amongst LTFT trainees. Those returning as full-time trainees reported feeling it took roughly 3 weeks for their confidence to return.

As a result of the shortcomings in the RTW process highlighted by this survey a Return to Work Programme was developed and put into practice in the West Midlands (see Chapter 4). Similar programmes have been established in other LETBs.

Following the introduction of the RTW programme the survey was repeated in 2015 and on this occasion consultants known to have had time off were also surveyed. The results of this second survey indicated some improvements. The trainee responses demonstrated increased awareness and uptake of KIT days; in addition many trainees were using the RTW programme. Overall it appeared that fewer problems were encountered with returning to work and the length of time reported to regain confidence had reduced in variability and length, with the longest estimate being 3 months (compared to the previously reported 6 months).

The consultants' survey indicated some (but poorer overall) uptake of the RTW programme and increased variability in estimated time to return of confidence (days to 6 months). Unsurprisingly common concerns among consultants returning to work highlighted some different areas, which included ability to support trainees and awareness of human factors. It was noted a higher proportion of consultants take career breaks due to sick compared to maternity leave and a concern in this group was having the stamina to work through the day and worries about fatigue. In both trainee and consultant groups less than 
$50 \%$ of individuals had a mentor and the vast majority indicated they would have liked this support.

Other interesting comments included one from a trainee who thought after taking her third period of leave returning to work would become easier when actually she found it was more difficult because of the responsibilities of becoming more senior. One consultant noted that they felt reassured as everything came back very quickly, which they attributed to having been doing it for a long time before they went off.

There are an increasing number of publications providing guidance to smooth individuals' transitions from prolonged periods of leave back into clinical anaesthesia. These aim to make the process as clear and stress free for those returning to work as possible. In the case of RTW programmes this is done by laying out some requirements thereby ensuring some of the responsibility for appropriate reintroduction to the clinical environment lies with the employers. Ultimately, however, the responsibility remains with the individual to prepare for and plan a reintroduction to work. Many of the resources available to help with this are described and illustrated in the following chapters.

\section{References}

1. L. Jobling. Returning to work - a personal view. RCOA Bulletin. 2011; 66: 29-31.

2. E. V. E. Plunkett, C. L. Baxendale, N. Osborn et al. Returning to work: a survey of recent trainee experience and introduction of a return to work programme. Anaesthesia 2013; 68: 991. 